\title{
High Rate Resistive Plate Chamber for LHC detector upgrades
}

\author{
Y. Haddad ${ }^{\text {a,* }}$, I. Laktineh ${ }^{\text {b }}$, G. Grenier ${ }^{\text {b }}$, N. Lumb ${ }^{\text {b }}$, S. Cauwenbergh ${ }^{\text {c }}$ \\ ${ }^{a}$ Laboratoire Leprince-Ringuet (LLR), École Polytechnique, 91120 Palaiseau, France \\ ${ }^{b}$ IPNL, Villeurbanne 69622 Lyon, France \\ ${ }^{c}$ Ghent University, Ghent, Belgium
}

\begin{abstract}
The limitation of the detection rate of standard bakelite resistive plate chambers (RPC) used as muon detectors in the LHC experiments has prevented the use of such detectors in the high rate regions in both CMS and ATLAS detectors. One alternative to these detectors are RPCs made with low resistivity glass plates $\left(10^{10} \Omega . \mathrm{cm}\right)$, a beam test at DESY has shown that such detectors can operate at few thousand $\mathrm{Hz} / \mathrm{cm}^{2}$ with high efficiency $(>90 \%)$.
\end{abstract}

Key words: Gaseous detectors, GRPC, High Rate Detectors PACS: 29.40.Cs, 29.40.Gx, 29.40.Vj

\section{Introduction}

RPCs are powerful detectors used in many HEP physics experiments. Their good time resolution and efficiency, in addition to their simplicity and low cost make them excellent candidates for very large area detectors. The high resistivity of glass plates helps to prevent discharge damage in these detectors, but this feature represents a weakness when it comes to their use in high rate environments.

A semi-conductive glass RPC (GRPC) is a solution to overcome this issue. The low resistivity of its doped glass accelerates the absorption of the avalanche's charge created when a charged particles crosses the RPC. A recent beam test at DESY in January 2012 with a high rate electron beam constitutes a validation of this new concept.

The GRPC detector is based on the ionization produced by charged particles in a gas gap. A typical gas mixture is $93 \%$ $\operatorname{TFE}\left(\mathrm{C}_{2} \mathrm{~F}_{4}\right), 5 \% \mathrm{CO}_{2}$ and $2 \% \mathrm{SF}_{6}$, contained in a $1.2 \mathrm{~mm}$ gap between 2 glass plates. A high voltage between $6.5 \mathrm{kV}$ and - $8 \mathrm{kV}$ was applied on the glass through a resistive coating, assuring the charge multiplication of initial ionizations in avalanche $\checkmark$ mode with a typical gain of $10^{7}$. The new aspect of this detector is the low resistivity of the doped silicate glass (less $>$ then $10^{10-11} \Omega$.cm, compared to the $10^{13} \Omega$.cm typical of float glass), provided by Tsinguha University following a new process [1]. The glass plate thickness is $1.1 \mathrm{~mm}$ for the cathode and $0.7 \mathrm{~mm}$ for the anode. The resistive coating is colloidal graphite of $1 M \Omega / \square$ resistivity. The gas was uniformly distributed in the chamber using the channeling-based system. Ceramic balls with $1.2 \mathrm{~mm}$ diameter were used as spacers. The total GRPC thickness was $3 \mathrm{~mm}$. The signal was collected by $1 \times 1 \mathrm{~cm}^{2}$ copper pads (figure $1 \mathrm{c}$ connected to a semi-digital readout system with 3 thresholds, identical to the one equipping the GRPC

\footnotetext{
${ }^{*}$ Corresponding author

Email addresses: haddad@llr.in2p3.fr (Y. Haddad)
}

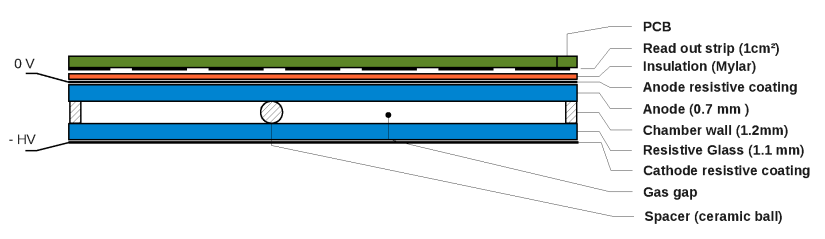

(a)

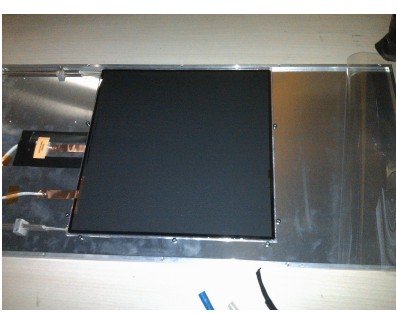

(b)

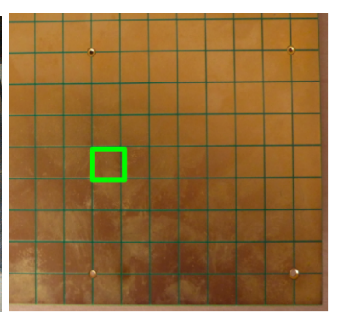

(c)
Figure 1: (a) Schematic drawing of GRPC with electrodes made of silicate glass. (b) photo of $30 \mathrm{~cm} \times 30 \mathrm{~cm}$ GRPC with semi-conductive glass. (c) readout pad with size of $1 \times 1 \mathrm{~cm}^{2}$

chambers used in the SDHCAL prototype developed within the CALICE collaboration [2][3].

\section{DESY test beam}

Four $30 \times 30 \mathrm{~cm}^{2}$ area RPCs were built following the design shown in figure 1a and were tested at DESY in January 2012. The DESY II synchrotron provides an intense and continuous electron beam with an energy up to $6 \mathrm{GeV}$. The particle rate depends on the beam energy, with a maximum of $35 \mathrm{kHz}$. The beam size is a few $\mathrm{cm}^{2}$. Two scintillator detectors were placed upstream of the detector. Their role was to measure the beam 
rate. One additional GRPC made with standard glass was added

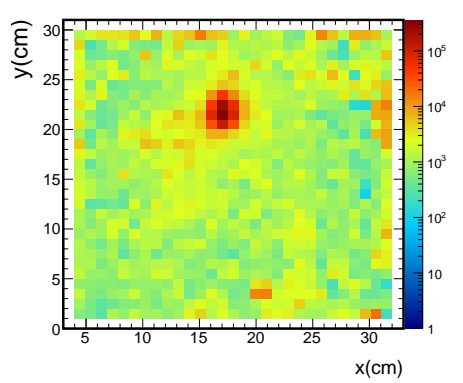

Figure 2: Beam profile in the chambers with $e^{-}$at $2 \mathrm{GeV}$.

to the setup.

\section{Results \& discussion}

\subsection{GRPC performances}

The local efficiency and multiplicity were measured by using 3 chambers to reconstruct particle tracks and determining the expected hit position in the 4 th. The multiplicity $\mu$ is defined as the number of fired pads within $3 \mathrm{~cm}$ of the expected position. The efficiency $\epsilon$ is the fraction of tracks with $\mu \geq 1$. The efficiency (3a) and multiplicity (3b) were measured as function of the polarization high voltage. The same threshold was used for all voltages. The threshold value is fixed at $50 \mathrm{fC}$ and $7.2 \mathrm{kV}$ was chosen as the working point, giving $(\mu, \epsilon)=(1.4,95 \%)$.

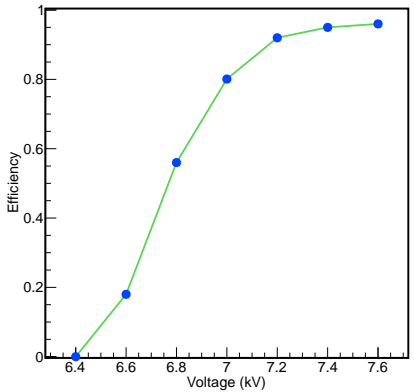

(a)

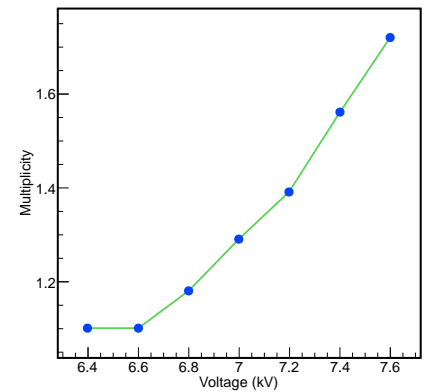

(b)
Figure 3: (a) Efficiency vs high voltage scan. (b) Multiplicity vs high voltage scan

\subsection{Running in a high rate beam}

The scintillator detectors were used to determine the total particle flux, which was then divided by the beam RMS area $\left(\approx 4 \mathrm{~cm}^{2}\right)$ to obtain the rate by unit area. The measured $(\mu, \epsilon)$ for different beam rates are plotted in figure 4 . The chamber with standard float glass (GRPC 1) becomes inefficient at rate exceeding one hundred $\mathrm{Hz} / \mathrm{cm}^{2}$ (above $1 \mathrm{kHz}$ the efficiency is below 10\%) while the semi-conductive chambers (GRPC 2-5) maintain a high efficiency $\geq 90 \%$ until at least $9 \mathrm{kHz} / \mathrm{cm}^{2}$.

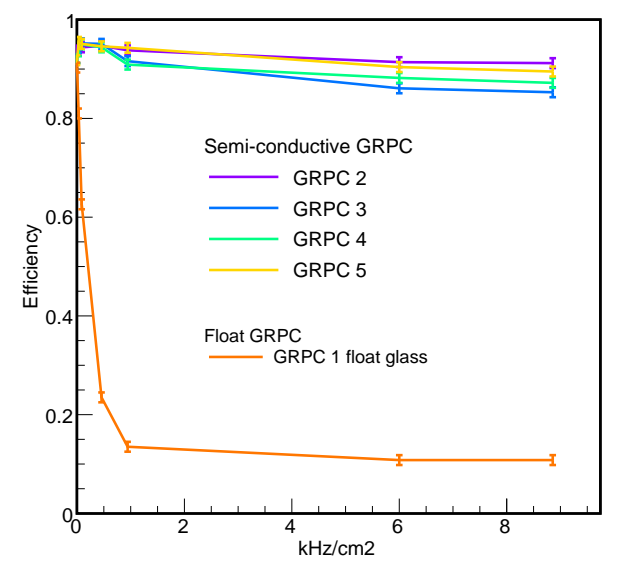

Figure 4: Efficiency vs rate for different RPC. The orange line correspond to the GRPC with float glass. The semi-conductive chambers are represented with different colors.

\section{Conclusion}

Semi conductive glass RPCs were tested at DESY in a high rate electron beam, producing very encouraging results; it has been shown that the main weakness of standard RPCs, namely the drop of efficiency at high rate, is clearly overcome, with efficiencies remaining at around $90 \%$ at rate of $9 \mathrm{kHz} / \mathrm{cm}^{2}$. This feature, combined with GRPC capability to provide precise time measurement, makes them an excellent candidate for future LHC muon detector upgrades. Additional studies on their aging under high rate conditions are underway. A multigap version is also under investigation.

\section{References}

[1] Wang, Yi; Wang, Jingbo; Yan, Qiang; Li, Yuanjing; Cheng, Jianping; , "Study on the performance of high rating MRPC," Nuclear Science Symposium Conference Record, 2008. NSS '08. IEEE , vol., no., pp.913-916, 19-25 Oct. 2008 doi: 10.1109/NSSMIC.2008.4774543

[2] Kieffer, R.; Laktineh, I.B.; Lumb, N.; Bedjidian, M.; Donckt, M.V.; Han, R.; Mirabito, L.; , 'Development of new kind of GRPC for a semi-digital hadronic calorimeter," Nuclear Science Symposium Conference Record (NSS/MIC), 2010 IEEE , vol., no., pp.1468-1471, Oct. 30 2010-Nov. 6 2010 doi: 10.1109/NSSMIC.2010.5874016

[3] Laktineh, I.; , "Development of a semi-digital hadronic calorimeter using GRPCs for future linear collider experiments," Nuclear Science Symposium Conference Record (NSS/MIC), 2009 IEEE, vol., no., pp.100-102, Oct. 24 2009-Nov. 12009 doi: 10.1109/NSSMIC.2009.5401858

[4] AIDA DESY II

http://adweb.desy.de/\$\sim\$testbeam/ 